\title{
iPSC-derived mesenchymal stromal cells are less supportive than primary MSCs for co- culture of hematopoietic progenitor cells
}

\author{
Theresa Vasko ${ }^{1,2+}$, Joana Frobel ${ }^{1,2 \dagger}$, Richard Lubberich ${ }^{1,2}$, Tamme W. Goecke $^{3}$ and Wolfgang Wagner ${ }^{1,2^{*}}$
}

\begin{abstract}
In vitro culture of hematopoietic stem and progenitor cells (HPCs) is supported by a suitable cellular microenvironment, such as mesenchymal stromal cells (MSCs) — but MSCs are heterogeneous and poorly defined. In this study, we analyzed whether MSCs derived from induced pluripotent stem cells (iPS-MSCs) provide a suitable cellular feeder layer too. iPSMSCs clearly supported proliferation of HPCs, maintenance of a primitive immunophenotype $\left(\mathrm{CD} 34^{+}, \mathrm{CD} 133^{+}, \mathrm{CD} 38^{-}\right)$, and colony-forming unit (CFU) potential of CD34+ HPCs. However, particularly long-term culture-initiating cell (LTC-IC) frequency was lower with iPS-MSCs as compared to primary MSCs. Relevant genes for cell-cell interaction were overall expressed at similar level in MSCs and iPS-MSCs, whereas VCAM1 was less expressed in the latter. In conclusion, our iPSMSCs support in vitro culture of HPCs; however, under the current differentiation and culture conditions, they are less suitable than primary MSCs from bone marrow.
\end{abstract}

We followed the hypothesis that iPS-MSCs might provide an unlimited and more standardized alternative to primary MSCs for stromal support of hematopoietic stem and progenitor cells (HPCs). To this end, we have reprogrammed bone marrow-derived MSCs into iPSCs and subsequently re-differentiated them towards iPS-MSCs as described before [1]. iPS-MSCs revealed similar fibroblastoid morphology, immunophenotype, and in vitro differentiation potential as primary MSCs (Additional file 1). HPCs were isolated from cord blood after written consent (Ethic Committee of RWTH Aachen: EK187/08). CD34 ${ }^{+}$cells were stained with carboxyfluorescein succinimidyl ester (CFSE) to monitor cell proliferation [2]. Flow cytometric analysis of residual CFSE staining after 5 days demonstrated that HPCs proliferated significantly faster if cultured with stromal support of either MSCs or iPS-MSCs (Fig. 1a). CD34 expression declined within a few cell divisions without feeder layer, whereas it was largely maintained over five subsequent cell divisions under both co-culture conditions

\footnotetext{
*Correspondence: wwagner@ukaachen.de

${ }^{\dagger}$ Equal contributors

${ }^{1}$ Helmholtz-Institute for Biomedical Engineering, Stem Cell Biology and Cellular Engineering, RWTH Aachen University Medical School, Pauwelsstrasse 20, 52074 Aachen, Germany

${ }^{2}$ Institute for Biomedical Engineering —Cell Biology, University Hospital of RWTH Aachen, Aachen, Germany

Full list of author information is available at the end of the article
}

(Fig. 1b). Overall, the expression of CD34 and CD133 declines after five cell divisions, which is consistent with previous observations [2]. Statistical analysis of CD34, CD38, CD45, and CD133 expression in relation to the cell division numbers indicated that co-culture with primary MSCs was slightly advantageous as compared to iPSMSCs for maintenance of a primitive hematopoietic immunophenotype (Fig. 1c).

We assessed the CFU frequency in freshly isolated HPCs or upon culture-expansion for 7 days: without stromal support, there was no expansion of CFUs, whereas CFU frequency was significantly increased under co-culture conditions with MSCs or iPS-MSCs (Fig. 1d). CFU frequency was not significantly affected if HPCs were co-cultured either with MSCs or iPS-MSCs, and there was no bias towards specific types of colonies (Fig. 1d). However, if HPCs were cultured for 5 weeks in a long-term culture-initiating cell (LTC-IC) assay [2], different hematopoiesis supporting capacities of MSCs and iPSMSCs became evident: long-term culture of HPCs gave rise to a significantly higher number of colonies on MSCs compared to iPS-MSCs (Fig. 1e).

There is evidence that besides cytokine secretion, direct cell-cell interaction between HPCs and MSCs is crucial for the hematopoiesis supportive function and 


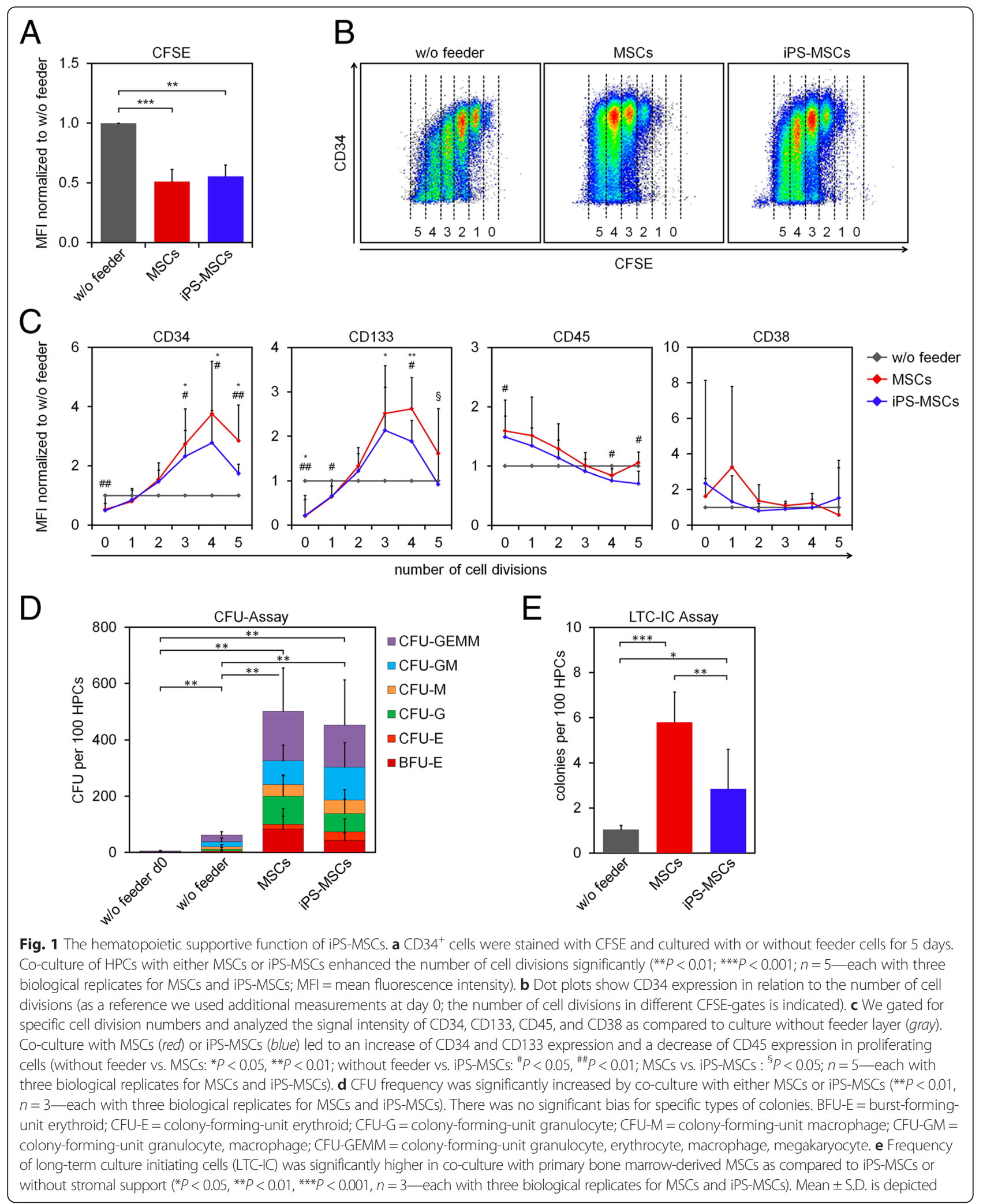

migration [3-5] -and this is reflected by cellular polarization $[6,7]$. In fact, co-culture with MSCs gave rise to a significantly higher fraction of elongated cells as compared to iPS-MSCs or feeder-free conditions (Fig. 2a). Subsequently, we reanalyzed previously published gene expression profiles of MSCs, iPSCs, and 


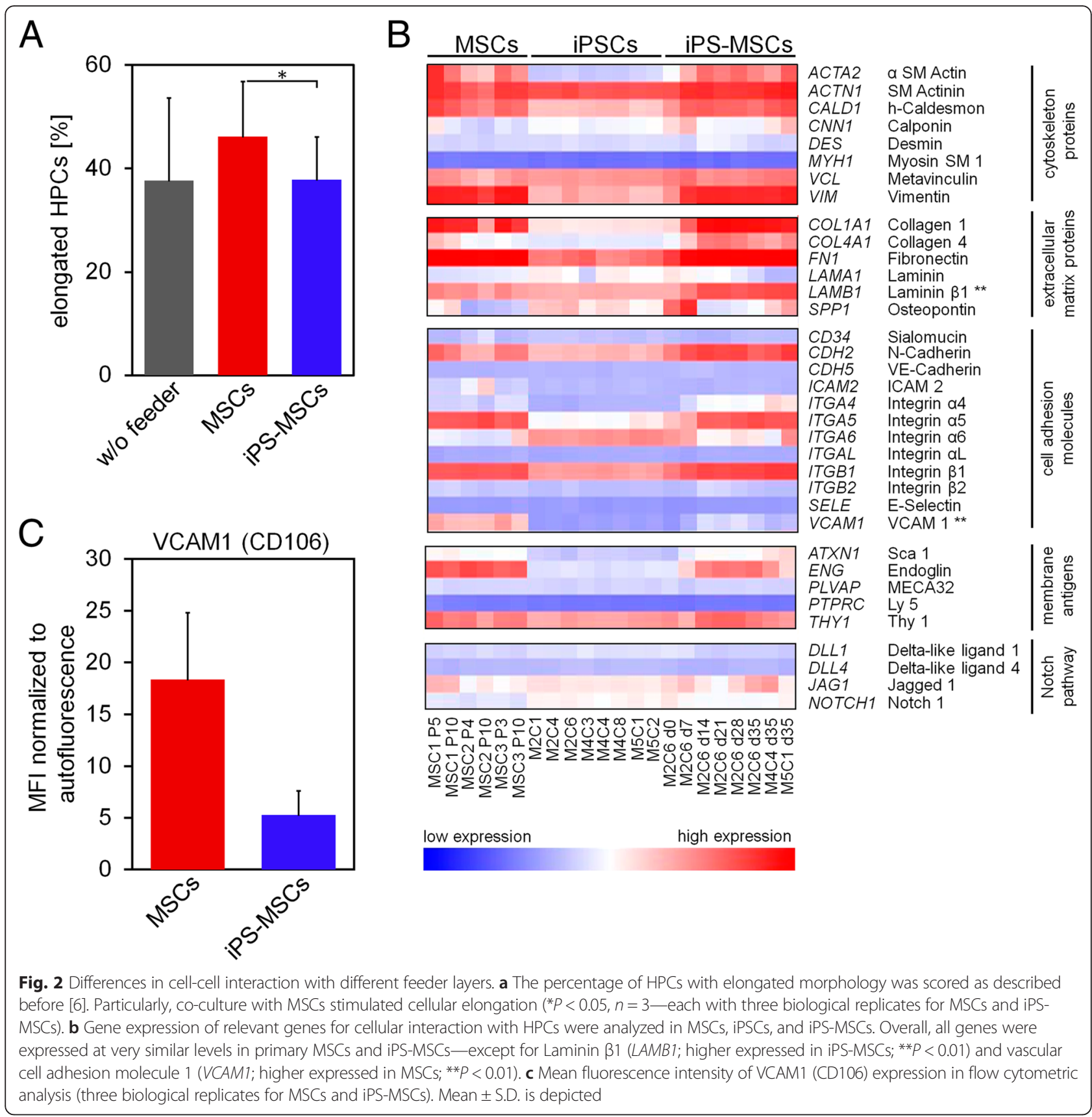

iPS-MSCs (GSE46019, GSE38806, and GSE54766) [1] with focus on a set of genes that has been considered to be functionally relevant for cell-cell interaction [8]. Overall, these genes were expressed at very similar levels in MSCs and iPS-MSCs, underlining the close molecular relationship of both cell preparations (Fig. 2b). Among the selected genes, only laminin $\beta 1$ (LAMB1) was higher expressed in iPS-MSCs (limma adjusted $P$ value: $P=0.004)$, and the vascular cell adhesion molecule 1 (VCAM1; CD106) was higher expressed in MSCs $(P=0.0018)$. This trend was also observed by flow cytometric analysis of VCAM1
(Fig. 2c), although it was only expressed in a relatively small subset of MSCs. We have previously demonstrated that VCAM1 is higher expressed in bone marrow-derived MSCs than in adipose tissue-derived MSCs [9]. Furthermore, we have shown that siRNAmediated knockdown of VCAM1 in MSCs entails lower proliferation rates of co-cultured HPCs [2]. It has been suggested that VCAM1 positive and negative subsets of MSCs differ in their biological function $[10,11]$ and that particularly the VCAM1 positive subset has higher immunoregulatory potential [11]. Lower expression of VCAM1 in iPS-MSCs might therefore be one reason 
for reduced stromal support. On the other hand, Ncadherin $(\mathrm{CDH} 2)$, which is also relevant for interaction of MSCs with HSCs [5], was in tendency higher expressed in iPS-MSCs than MSCs. Notably, differential expression of $L A M B 1, V C A M 1$, and $C D H 2$ was also reflected in DNA methylation patterns of MSCs and iPS-MSCs (Additional file 2). Either way, it is likely that a combination of adhesion proteins and chemokines evokes the differences in hematopoiesis supportive potential.

Taken together, our iPS-MSCs provide a less hematopoiesis supportive microenvironment than primary MSCs, particularly after long-term co-culture. This tendency was not observed in a recent study by Moslem et al. [12], but these authors did not test for maintenance of LTC-ICs. It is conceivable that optimized differentiation procedures of iPSCs towards cellular components of the hematopoietic stem cell niche as well as 3D-culture systems will further enhance stromal support to ultimately facilitate in vitro expansion of HPCs.

\section{Additional files}

Additional file 1: Functional characterization of iPS-MSCs. (A) Phase contrast images of MSCs and iPS-MSCs: iPS-MSCs revealed similar fibroblastoid morphology as MSCs. (B) iPS-MSCs displayed similar immunophenotypic characteristics as primary MSCS (MFI = mean fluorescence intensity; mean \pm S.D. of three biological replicates is presented; ${ }^{*} P<0.05,{ }^{*} P<0.01$ ). (C) MSCs and iPS-MSCs were differentiated for three weeks towards adipogenic, osteogenic, and chondrogenic lineages and subsequently stained with BODIPY/DAPI, Alizarin Red, or Alcian Blue/PAS, respectively (in analogy to our previous work [1]). Controls were simultaneously cultured in normal growth medium (DMEM supplemented with $10 \%$ human platelet lysate). Representative images are shown. (PDF $153 \mathrm{~kb}$ )

Additional file 2: DNA methylation is in line with differential expression of VCAM1, CDH2, and LAMB1. DNA methylation levels of CpG dinucleotides in the genes VCAM1, CDH2, and LAMB1 were analyzed for bone marrow-derived MSCs, iPS-MSCs, and iPSCs using the Illumina $450 \mathrm{k}$ BeadChip data (GSE17448 and GSE54767) as described in detail in our previous work [1]. DNA methylation level is given as $\beta$-value ranging from 0 (no methylation) to 1 (100\% methylation). Genomic location of the respective CpG sites and statistical significance of MSCs vs. iPS-MSCs are indicated $\left({ }^{*} P<0.05,{ }^{* *} P<0.01,{ }^{* * *} P<0.001\right.$, TSS $1500=1500$ bp upstream of transcription start site; TSS200 $=200$ bp upstream of TSS; UTR $=$ untranslated region). DNA methylation of VCAM1 was higher in iPS-MSCS than MSCs. In contrast, close to the transcription start site of $\angle A M B 1$ and $C D H 2$ several CpGs revealed significantly lower DNA methylation in iPS-MSCs than primary MSCs. These epigenetic differences may therefore be relevant for the observed differences in gene expression. (PDF $362 \mathrm{~kb}$ )

\section{Competing interests}

The authors declare that they have no competing interests.

\section{Authors' contributions}

TV and JF differentiated the iPSCs towards MSCs, performed the co-culture experiments, analyzed the microarray data, and drafted the manuscript. RL performed the LTC-IC assays. TWG provided the materials and helped in the study design. WW conceived the study and participated in its design, data analysis, coordination, and drafting of the manuscript. All authors read and approved the final manuscript.

\section{Acknowledgements}

This work was supported by the Else Kröner-Fresenius Stiftung (2014_A193), by the German Research Foundation (WA/1706/2-1), and by the Interdisciplinary Center for Clinical Research (IZKF) in the Faculty of Medicine at the RWTH Aachen University (O1-1).

\section{Author details}

${ }^{1}$ Helmholtz-Institute for Biomedical Engineering, Stem Cell Biology and Cellular Engineering, RWTH Aachen University Medical School, Pauwelsstrasse 20, 52074 Aachen, Germany. ${ }^{2}$ Institute for Biomedical Engineering-Cell Biology, University Hospital of RWTH Aachen, Aachen, Germany.

${ }^{3}$ Department of Obstetrics and Gynecology, RWTH Aachen University Hospital, Aachen, Germany.

Received: 2 March 2016 Accepted: 13 April 2016

Published online: 21 April 2016

\section{References}

1. Frobel J, Hemeda H, Lenz M, Abagnale G, Joussen S, Denecke B, Saric T, Zenke M, Wagner W. Epigenetic rejuvenation of mesenchymal stromal cells derived from induced pluripotent stem cells. Stem Cell Reports. 2014;3:41422.

2. Walenda T, Bork S, Horn P, Wein F, Saffrich R, Diehlmann A, Eckstein V, Ho AD, Wagner W. Co-culture with mesenchymal stromal cells increases proliferation and maintenance of hematopoietic progenitor cells. J Cell Mol Med. 2010;14:337-50.

3. Jing D, Fonseca AV, Alakel N, Fierro FA, Muller K, Bornhauser M, Ehninger G, Corbeil D, Ordemann R. Hematopoietic stem cells in co-culture with mesenchymal stromal cells_-modeling the niche compartments in vitro. Haematologica. 2010;95:542-50.

4. Wagner W, Saffrich R, Wirkner U, Eckstein V, Blake J, Ansorge A, Schwager C, Wein F, Miesala K, Ansorge W, Ho AD. Hematopoietic progenitor cells and cellular microenvironment: behavioral and molecular changes upon interaction. Stem Cells. 2005:23:1180-91.

5. Wein F, Pietsch L, Saffrich R, Wuchter P, Walenda T, Bork S, Horn P, Diehlmann A, Eckstein V, Ho AD, Wagner W. N-cadherin is expressed on human hematopoietic progenitor cells and mediates interaction with human mesenchymal stromal cells. Stem Cell Res. 2010;4:129-39.

6. Wagner W, Ansorge A, Wirkner U, Eckstein V, Schwager C, Blake J, Miesala K, Selig J, Saffrich R, Ansorge W, Ho AD. Molecular evidence for stem cell function of the slow-dividing fraction among human hematopoietic progenitor cells by genome-wide analysis. Blood. 2004;104:675-86.

7. Giebel B, Corbeil D, Beckmann J, Hohn J, Freund D, Giesen K, Fischer J, Kogler G, Wernet P. Segregation of lipid raft markers including CD133 in polarized human hematopoietic stem and progenitor cells. Blood. 2004;104: 2332-8.

8. Charbord P, Oostendorp R, Pang W, Herault O, Noel F, Tsuji T, Dzierzak E, Peault B. Comparative study of stromal cell lines derived from embryonic, fetal, and postnatal mouse blood-forming tissues. Exp Hematol. 2002;30: 1202-10.

9. Wagner W, Roderburg C, Wein F, Diehlmann A, Frankhauser M, Schubert R, Eckstein $V$, Ho AD. Molecular and secretory profiles of human mesenchymal stromal cells and their abilities to maintain primitive hematopoietic progenitors. Stem Cells. 2007;10:2638-57.

10. Mabuchi Y, Morikawa S, Harada S, Niibe K, Suzuki S, Renault-Mihara F, Houlihan DD, Akazawa C, Okano H, Matsuzaki Y. LNGFR(+)THY-1(+)VCAM$1(\mathrm{hi}+)$ cells reveal functionally distinct subpopulations in mesenchymal stem cells. Stem Cell Reports. 2013;1:152-65.

11. Yang ZX, Han ZB, Ji YR, Wang YW, Liang L, Chi Y, Yang SG, Li LN, Luo WF, Li JP, Chen DD, Du WJ, Cao XC, Zhuo GS, Wang T, Han ZC. CD106 identifies a subpopulation of mesenchymal stem cells with unique immunomodulatory properties. PLoS One. 2013;8:e59354.

12. Moslem M, Eberle I, Weber I, Henschler R, Cantz T. Mesenchymal stem/stromal cells derived from induced pluripotent stem cells support CD34(pos) hematopoietic stem cell propagation and suppress inflammatory reaction. Stem Cells Int. 2015;2015:843058. 